Protein engineering

\title{
Hydrogen bonds in active sites
}

\author{
from John Galloway
}

SINCE the sceptical reception of Latimer and Rodebush's suggestion in 1920 that they hold water molecules together, hydrogen bonds have come a long way. They are now widely believed to play the definitive role in determining molecular specificity. Hence the interest of protein engineers in hydrogen bonds and the paper on page 235 of this issue in which Alan Fersht and colleagues analyse, by means of systematically altering the amino acids of the active site of an enzyme, the hydrogen bonds that determine substrate specificity.

Full realization of the potential of protein engineering is some way off. The chief problem is our inexact understanding of the energetics of the three-dimensional structures of proteins and of the relationships between those structures and the proteins' functions. It is well enough established that the sequence of amino acids uniquely defines a protein's three-dimensional structure and, in doing so, confers on it a capacity to interact functionally with perhaps only one other molecule. Driven to the molecular level, the study of biological specificity thus becomes the detailed analysis of this structural and functional idiosyncracy and the mechanisms by which it is achieved.

Without a detailed understanding of the way proteins are put together, it is inconceivable that new proteins can be designed or old proteins given new roles. But that understanding is hard to win in the degree of detail needed, and the capacity to determine structure and function by protein crystallography still far outstrips any attempt to predict it from amino-acid sequence. It may become possible to mimic protein folding by analogue means but otherwise we are left with energy calculations which are not easy. Although, among other things, fair calculations can be done 'in vacuo', proteins fold in water, which probably mediates all interactions of any interest. Since it is now possible to change any residue in a protein by site-directed mutagenesis of its gene, the present compromise is to study empirically the structural and energetic changes associated with small changes in amino-acid sequences.

For Fersht et $a l$., the protein under study is the enzyme tyrosyl-transfer RNA synthetase which activates tyrosine, forming a complex between the enzyme and the intermediate tyrosyl-adenylate, before transferring the amino acid to its transfer RNA. (The enzyme is of no apparent medical or commercial interest.) The power of the approach lies in combining site-directed mutagenesis both with enzyme kinetics, which gives accurate estimates of changes in binding energies, and with highresolution crystallography, which gives the detailed structural changes. (Without high-resolution crystallography the technique can still be used, but merely as a probe of the role of residues in catalysis, for instance.) A logistical difficulty that such combined methodology usually has to face is that the techniques of mutagenesis and kinetics are quick to yield results, but protein crystallography tends to lag behind. It is striking that this is not the case for the tyrosyl-transfer RNA synthetase studies, presumably in part because of growing familiarity with its structure.

The most noteworthy of a number of findings described by Fersht $e t a l$. is that a hydrogen bond involving a side chain with a net charge on it is stronger - by up to a factor of about six - than a hydrogen bond with no such involvement. Indeed by the time the difference in energy is properly weighted by a Boltzman exponential factor, a 'charged' hydrogen bond may well contribute one to two orders of mag- nitude more to specificity than an 'uncharged' one. That is useful to know in building up a database of interactions and it also reminds us of something that is often forgotten - hydrogen bonds are to a large extent simply a convenient way of looking at a part of the overall spectrum of electrostatic interactions between molecules or parts of molecules. A convenient way of expressing such an interaction is in terms of a multipole expansion

$$
\frac{e_{1} e_{2}}{r_{12}}+\frac{e_{1} \mu_{2}+e_{2} \mu_{1}}{r_{12}{ }^{2}}+\frac{\mu_{1} \mu_{2}}{r_{12}{ }^{3}}+\cdots \cdots \cdot
$$

where $e$ represents net charge and $\mu$ a dipole and 1,2 refer to the interacting groups. The leading term in computing a hydrogen bond between neutral groups - like water molecules - is the third one. But if one group has a net charge, then the second term will lead and typically will be a good deal bigger. Since the problem is complicated by the presence of strongly dipolar water molecules, it would not have been very surprising had the effect of the charged hydrogen bond not shown up. That it has done, is well worth noting. $\square$

John Galloway is at the Medical Research Council, 20 Park Crescent, London WIN $4 A L$, UK.

\section{Ecology}

\section{Competition in imaginary worlds}

\section{from Paul H. Harvey and Robert M. May}

To WHAT extent does competition influence the distribution of species among the islands of an archipelago? This seemingly straightforward question cannot easily be answered despite scores of studies using a variety of techniques to analyse the distributions of many plant and animal species from several groups of islands ${ }^{1}$. No consistent patterns emerge. Indeed, various authors working on the same data set often reach different conclusions because they have used different assumptions in their statistical models. A new approach by Colwell and Winkler ${ }^{2}$, using a computer to generate a myriad of imaginary worlds, helps us to understand the limitations of conventional analyses for detecting the factors that actually govern the distribution of species in archipelagos.

The essential problem is that each archipelago is unique, with its plant and animal species put in place by the slow workings of evolutionary processes. The logically appealing techniques of The Scientific Method - manipulative experiments with controls and replicates - are not easily applied in the real world. As emphasized by Simberloff, Strong and others at Florida State University ${ }^{3}$, one way of trying to tease out the processes underlying observed patterns of distributions of species is to compare these patterns with null models constructed by randomly reshuffling the species among the islands. But the effects of competition may still inadvertantly be built into the null models, which assume that competition is absent

Colwell and Winkler ${ }^{2}$ shed light on these problems by generating imaginary archipelagos, each populated according to specified rules. Using the worlds they have created, they explore the extent to which conventional methods of analysis can detect the competitive or other factors that actually govern the distribution of species in their archipelagos.

They begin by generating phylogenies, using a simple simulation model designed by Raup and Gould ${ }^{4}$. As the hypothetical species evolve, their phenotypes change gradually so that, on average, closely related species are more like each other than are more distantly related species. For the purposes of the model, easily measurable characters such as beak length and width are chosen. This evolution takes place on a large land mass under the guidance of a computer program called GOD. The organisms then disperse to the islands of a nearby archipelago, their destinations determined by additional computer routines, summarized in the figure.

One routine allows random invasion of the different islands with subsequent interspecific competition determining which species establish successful populations on each island. When phenotypically similar species invade an 